PROCEEDINGS OF THE

AMERICAN MATHEMATICAL SOCIETY

Volume 137, Number 1, January 2009, Pages 359-369

S 0002-9939(08)09455-0

Article electronically published on August 26, 2008

\title{
SUSPENSION THEOREMS FOR LINKS AND LINK MAPS
}

\author{
MIKHAIL SKOPENKOV
}

(Communicated by Alexander N. Dranishnikov)

\begin{abstract}
We present a new short proof of the explicit formula for the group of links (and also link maps) in the "quadruple point free" dimension. Denote by $L_{p, q}^{m}$ (respectively, $C_{p}^{m-p}$ ) the group of smooth embeddings $S^{p} \sqcup S^{q} \rightarrow S^{m}$ (respectively, $S^{p} \rightarrow S^{m}$ ) up to smooth isotopy. Denote by $L M_{p, q}^{m}$ the group of link maps $S^{p} \sqcup S^{q} \rightarrow S^{m}$ up to link homotopy.

Theorem 1. If $p \leq q \leq m-3$ and $2 p+2 q \leq 3 m-6$, then

$L_{p, q}^{m} \cong \pi_{p}\left(S^{m-q-1}\right) \oplus \pi_{p+q+2-m}\left(S O / S O_{m-p-1}\right) \oplus C_{p}^{m-p} \oplus C_{q}^{m-q}$.

Theorem 2. If $p, q \leq m-3$ and $2 p+2 q \leq 3 m-5$, then $L M_{p, q}^{m} \cong \pi_{p+q+1-m}^{S}$.

Our approach is based on the use of the suspension operation for links and link maps, and suspension theorems for them.
\end{abstract}

\section{INTRODUCTION}

This paper is on the knotting problem of higher-dimensional manifolds (for recent surveys see [21, 24]). We study knots and links in codimension at least 3, where a complete answer can sometimes be obtained, in contrast to the classical situation of simple closed curves in $\mathbb{R}^{3}$.

Denote by $L_{p, q}^{m}$ the set of smooth embeddings $S^{p} \sqcup S^{q} \rightarrow S^{m}$ up to smooth isotopy. Denote by $C_{p}^{m-p}$ the set of smooth embeddings $S^{p} \rightarrow S^{m}$ up to smooth isotopy. For $p, q \leq m-3$ these sets are commutative groups with respect to the "componentwise connected sum" operation [8].

The main result of this paper is a new short proof of an explicit formula for the group $L_{p, q}^{m}$ in terms of the groups $C_{p}^{m-p}, C_{q}^{m-q}$ and certain homotopy groups:

Theorem 1.1. If $p \leq q \leq m-3$ and $2 p+2 q \leq 3 m-6$, then

$$
L_{p, q}^{m} \cong \pi_{p}\left(S^{m-q-1}\right) \oplus \pi_{p+q+2-m}\left(V_{M+m-p-1, M}\right) \oplus C_{p}^{m-p} \oplus C_{q}^{m-q} .
$$

Here $V_{M+l, M}$ is the Stiefel manifold of $M$-frames at the origin of $\mathbb{R}^{M+l}$, where $M$ is large. Many of the groups $\pi_{n}\left(V_{M+l, M}\right)$ and $C_{p}^{m-p}$ are known 20, 7].

Example 1.2. $L_{3,3}^{6} \cong \mathbb{Z} \oplus \mathbb{Z} \oplus \mathbb{Z} \oplus \mathbb{Z}$.

Received by the editors May 15, 2006, and, in revised form, November 1, 2007.

2000 Mathematics Subject Classification. Primary 57Q45, 57R40; Secondary 55P40, 57Q30.

Key words and phrases. Link, link map, link homotopy, homotopy groups, Stiefel manifold, suspension, the EHP sequence, engulfing, linking number, alpha-invariant, beta-invariant.

The author was supported in part by INTAS grant 06-1000014-6277, Russian Foundation of Basic Research grants 05-01-00993-a, 06-01-72551-NCNIL-a, 07-01-00648-a, President of the Russian Federation grant NSh-4578.2006.1, Agency for Education and Science grant RNP-2.1.1.7988, and Moebius Contest Foundation for Young Scientists.

(C)2008 American Mathematical Society Reverts to public domain 28 years from publication 
Theorem 1.1 is the strongest available explicit classification of 2-component links in spheres. However, for arbitrary $p, q \leq m-3$ there is a famous exact sequence involving the groups $L_{p, q}^{m}$, certain homotopy groups and maps between them involving Whitehead products (see [8, Theorem 1.1] and [5]).

Theorem 1.1] was proved in [8, Theorems 10.7 and 2.4] (under the stronger restrictions $p \leq q$ and $p+3 q \leq 3 m-7$; however, the Haefliger argument can be extended to cover our dimension range (see Remark 3.7)). The second inequality in Theorem 1.1 is sharp (see Remark 3.6).

We reduce the classification of links to the classification of link maps, which is an interesting problem in itself [22, 13, 6].

A link map is a continuous map $f: X \sqcup Y \rightarrow Z$ such that $f X \cap f Y=\emptyset$. A link homotopy is a continuous family of link maps $f_{t}: X \sqcup Y \rightarrow Z$. Denote by $L M_{p, q}^{m}$ the set of link maps $S^{p} \sqcup S^{q} \rightarrow S^{m}$ up to link homotopy. For $p, q \leq m-3$ this set is a commutative group with respect to the "componentwise connected sum" operation (by [22, p. 187], [12, Remark 2.4] and the "link concordance implies link homotopy" theorem cited below).

The second result of this paper is a short proof of the following theorem:

Theorem 1.3 ([6]). If $p, q \leq m-3$ and $2 p+2 q \leq 3 m-5$, then $L M_{p, q}^{m} \cong \pi_{p+q-m+1}^{S}$.

The isomorphism is the $\alpha$-invariant (see $\$ 3$ ). The second inequality is sharp 6$]$.

Theorem 1.3 is the strongest known explicit classification of link maps for $p, q \leq$ $m-3$. However, under a slightly weaker dimension restriction there is an exact sequence involving the groups $L M_{p, q}^{m}$ and certain bordism groups [13, Theorem A].

Our approach is based on the use of the suspension map. The suspension map $\Sigma: L M_{p, q}^{m} \rightarrow L M_{p+1, q}^{m+1}$ is defined by suspending the $p$-component and including the $q$-component. It is easy to see that for $M$ large, $L M_{p+M, q}^{m+M} \cong \pi_{p+q-m+1}^{S}$ [12. Thus Theorem 1.3 follows from the following assertion:

Theorem 1.4 (Suspension theorem for link maps 6]). If $p, q \leq m-3$, then the suspension map is bijective for $2 p+2 q \leq 3 m-5$ and surjective for $2 p+2 q \leq 3 m-4$.

This theorem had been known earlier only as a corollary of Theorem 1.3. We give a short direct proof of Theorem 1.4 analogous to Zeeman's proof of the higherdimensional Poincaré conjecture and using a version of Alexander's trick. Our proof is almost self-contained; we use only the "concordance implies isotopy" theorem.

Let us introduce some notions and conventions.

An embedding $f: X \times I \rightarrow S^{m} \times I$ is a concordance if $X \times 0=f^{-1}\left(S^{m} \times 0\right)$ and $X \times 1=f^{-1}\left(S^{m} \times 1\right)$. We tacitly use the facts that in codimension at least 3 concordance implies isotopy and any concordance or isotopy is ambient 9 .

Similarly, a link concordance is a continuous map $f:(X \sqcup Y) \times I \rightarrow S^{m} \times I$ such that $f(X \times I) \cap f(Y \times I)=\emptyset,(X \sqcup Y) \times 0=f^{-1}\left(S^{m} \times 0\right)$ and $(X \sqcup Y) \times 1=$ $f^{-1}\left(S^{m} \times 1\right)$. In codimension at least 3 link concordance implies link homotopy, which was announced in 17, discussed in 1, 16, 14, and proved in 18.

We say that a link map $f: S^{p} \sqcup S^{q} \rightarrow S^{m}$ is null link concordant if it extends to a link map $D^{p+1} \sqcup D^{q+1} \rightarrow D^{m+1}$. The latter link map is called null link concordance.

This paper is organized as follows. In $\$ 2$ we prove Theorem 1.4 In $\$ 3$ we deduce Theorem 1.1 from Theorem 1.4. Sections 2 and 3 can be read independently from each other.

In [2, 3] (cf. 23] ) a similar approach is applied to the classification of embeddings $S^{p} \times S^{q} \rightarrow S^{m}$. 


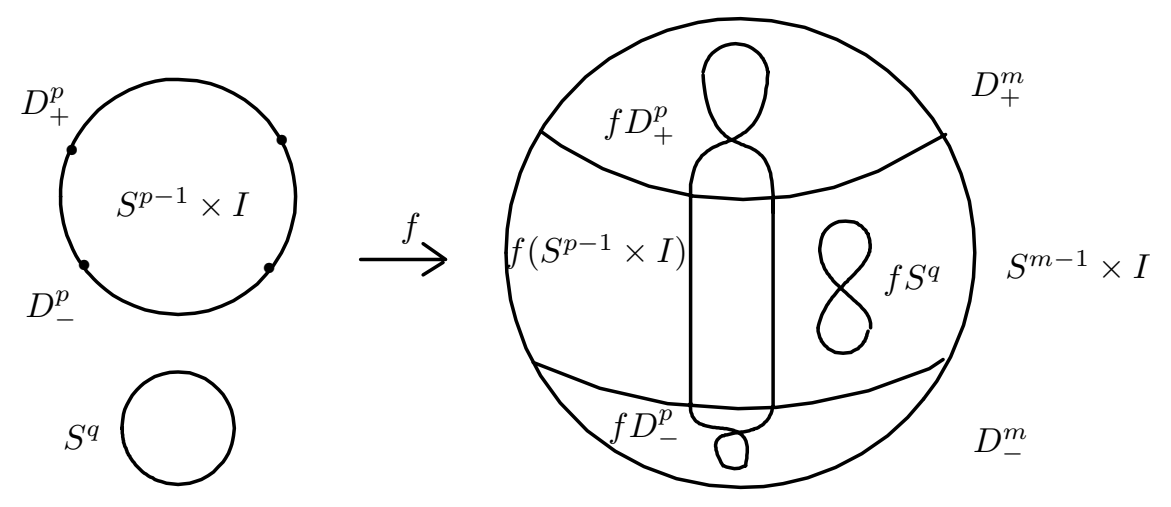

FiguRe 1. A standardized link map

\section{Classification of Link Maps}

We prove Theorem 1.4 as follows. First we prove the surjectivity in case $p \leq q$. Then we prove analogously the injectivity in case $p \leq q$, and finally we deduce the case $p>q$ of Theorem 1.4 from the case $p \leq q$.

Let us introduce our main notion and state our main lemma.

Definition 2.1 (see Figure 10. Let $S^{k}=D_{+}^{k} \cup\left(S^{k-1} \times I\right) \cup D_{-}^{k}$ be the standard decomposition of the sphere, where $\partial D_{+}^{k}=S^{k-1} \times 0=S^{k-1}$ is the equator of $S^{k}$. A link map $f: S^{p} \sqcup S^{q} \rightarrow S^{m}$ is standardized if the following three conditions hold:

(1) $f D_{+}^{p} \subset D_{+}^{m}, \quad f D_{-}^{p} \subset D_{-}^{m}, \quad f\left(S^{p-1} \times I\right) \subset S^{m-1} \times I$;

(2) $f S^{q} \subset S^{m-1} \times I$;

(3) $f\left(S^{p-1} \times I\right)$ is straight, i.e. $f\left(S^{p-1} \times I\right)=f\left(S^{p-1} \times 0\right) \times I$.

Lemma 2.2. Suppose that $p \leq q+1, p \leq m-3$ and $2 p+2 q \leq 3 m-5$; then any link map $f: S^{p} \sqcup S^{q} \rightarrow S^{m}$ is link homotopic to a standardized link map.

Proof of the surjectivity in Theorem 1.4 for $p \leq q$ modulo Lemma 2.2. Take a link map $f: S^{p+1} \sqcup S^{q} \rightarrow S^{m+1}$. Let us modify it to a suspension by a link homotopy.

By Lemma 2.2 we may assume that $f$ is standardized.

Push $f S^{q}$ along the fibers of $S^{m} \times I$ until it lies in $S^{m} \times 0=\partial D_{+}^{m+1}$. After that transform $f D_{+}^{p+1}$ and $f\left(S^{p+1}-\operatorname{Int} D_{+}^{p+1}\right)$ to the cones over $f \partial D_{+}^{p+1}$ in $D_{+}^{m+1}$ and in $S^{m+1}$ - Int $D_{+}^{m+1}$, respectively (by a rectilinear link homotopy). The obtained link map is the suspension of a link map $S^{p} \sqcup S^{q} \rightarrow S^{m}$.

Now we proceed to the proof of Lemma 2.2. First we prove it for $p \leq q$, then for $p=q+1$. The proofs of all technical claims below can be skipped for the first reading. From now until the end of $\$ 2$ we work in the piecewise linear category.

Proof of Lemma 2.2 for $p \leq q$. Let us make a generic link map $f: S^{p} \sqcup S^{q} \rightarrow S^{m}$ standardized by performing certain homeomorphisms $S^{p} \rightarrow S^{p}$ and $S^{m} \rightarrow S^{m}$. (Formally, performing homeomorphisms $h_{p}: S^{p} \rightarrow S^{p}$ and $h_{m}: S^{m} \rightarrow S^{m}$ means a link homotopy transforming $f$ to $h_{m} \circ f \circ\left(h_{p}^{-1} \sqcup \operatorname{Id}_{S^{q}}\right)$.)

(1) Construction of the homeomorphism $S^{p} \rightarrow S^{p}$ : splitting the sphere $S^{p}$. (The Zeeman engulfing; see Figure 2 on the left.) Consider the self-intersection set of 


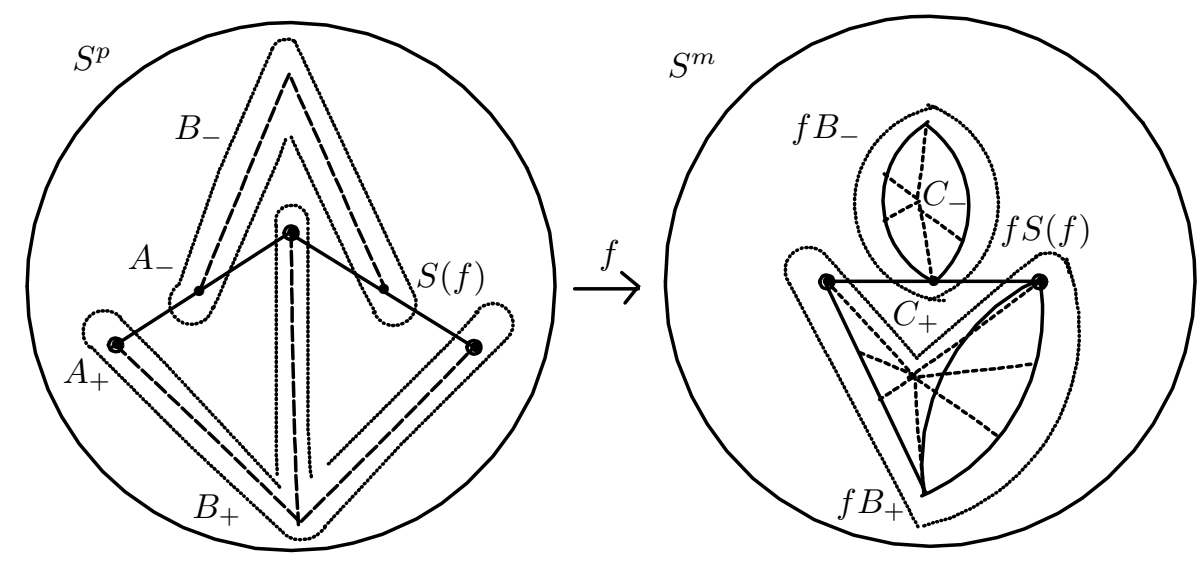

Figure 2. Splitting of the spheres $S^{p}$ and $S^{m}$

the $p$-component $S(f)=\operatorname{Cl}\left\{x \in S^{p}:\left|f^{-1} f x\right| \geq 2\right\}$. Let $A_{+}$be the skeleton of $S(f)$ formed by the simplices of dimension not greater than $\frac{1}{2} \operatorname{dim} S(f)$ (in a triangulation of $S^{p}, S^{q}$ and $S^{m}$ such that $f: S^{p} \sqcup S^{q} \rightarrow S^{m}$ is simplicial). Let $A_{-}$ be the subcomplex dual to $A_{+}$(i.e., $A_{-}$is the subcomplex formed by all simplices $\sigma$ of the first barycentric subdivision of $S(f)$ such that $\left.\sigma \cap A_{+}=\emptyset\right)$.

Claim 2.3. There exist subpolyhedra $B_{ \pm} \subset S^{p}$ such that $B_{ \pm} \supset A_{ \pm}$and $B_{ \pm} \cong C A_{ \pm}$.

Proof. Generically $\operatorname{dim} S(f) \leq 2 p-m$, so $\operatorname{dim} A_{ \pm} \leq p-\left[\frac{m+1}{2}\right]$. Take generic extensions $i_{ \pm}: C A_{ \pm} \rightarrow S^{p}$ of the inclusions $A_{ \pm} \hookrightarrow S^{p}$. They are embeddings, because $2\left(p-\left[\frac{m+1}{2}\right]+1\right)-p<0$ by the assumption $p \leq m-3$. Put $B_{ \pm}=i_{ \pm} C A_{ \pm}$.

Claim 2.4. Generically $B_{+} \cap B_{-}=\emptyset$ and $B_{ \pm} \cap S(f)=A_{ \pm}$.

Proof. This follows from $\operatorname{dim}\left(B_{+} \cap B_{-}\right) \leq 2\left(p-\left[\frac{m+1}{2}\right]+1\right)-p<0$ and $\operatorname{dim}\left(B_{ \pm}-A_{ \pm}\right) \cap S(f) \leq\left(p-\left[\frac{m+1}{2}\right]+1\right)+(2 p-m)-p \leq \frac{1}{2}(2 q+2 p-3 m+4)<0$, which is a corollary of the assumptions $p \leq q+1$ and $2 p+2 q \leq 3 m-5$.

Take disjoint regular neighborhoods of $B_{ \pm}$in $S^{p}$. Perform an orientationpreserving homeomorphism $S^{p} \rightarrow S^{p}$ taking these neighborhoods to the balls of the standard decomposition $S^{p}=D_{+}^{p} \cup\left(S^{p-1} \times I\right) \cup D_{-}^{p}$.

(2) Construction of the 1st homeomorphism $S^{m} \rightarrow S^{m}$ : splitting the sphere $S^{m}$. (See Figure 2 on the right.) Take subpolyhedra $C_{ \pm} \subset S^{m}$ such that $C_{ \pm} \supset f B_{ \pm}$ and $C_{ \pm} \cong C f B_{ \pm}$(constructed analogously to Claim 2.3 ).

Claim 2.5. Generically $C_{+} \cap C_{-}=\emptyset, C_{ \pm} \cap f S^{p}=f B_{ \pm}$and $C_{ \pm} \cap f S^{q}=\emptyset$.

Proof. This follows from the inequality $2\left(p-\left[\frac{m+1}{2}\right]+2\right)-m<0$, which holds by the assumption $p \leq m-3$, and $\operatorname{dim}\left(C_{ \pm}-f B_{ \pm}\right) \cap f S^{p} \leq\left(p-\left[\frac{m+1}{2}\right]+2\right)+p-m<0$, which holds by the assumptions $p \leq q$ and $2 p+2 q \leq 3 m-5$, and $\operatorname{dim}\left(C_{+} \cap f S^{q}\right) \leq$ $\left(p-\left[\frac{m+1}{2}\right]+2\right)+q-m<0$, which is equivalent to $2 p+2 q \leq 3 m-5$.

Take disjoint regular neighborhoods of $C_{ \pm} \cup f D_{ \pm}^{p}$ relatively $f \partial D_{ \pm}^{p}$ in $S^{m}-f S^{q}$. Perform a homeomorphism $S^{m} \rightarrow S^{m}$ taking them to the balls of the standard 
decomposition $S^{m}=D_{+}^{m} \cup\left(S^{m-1} \times I\right) \cup D_{-}^{m}$. By Claim 2.5 the obtained link map satisfies properties (11) and (2) of a standardized link map (see Definition 2.1).

To satisfy property (3) perform the following homeomorphism $S^{m} \rightarrow S^{m}$.

(3) Construction of the second homeomorphism $S^{m} \rightarrow S^{m}$ : straightening $f\left(S^{p-1} \times I\right)$.

Claim 2.6. There is a homeomorphism $S(f) \cap\left(S^{p-1} \times I\right) \cong\left(S(f) \cap\left(S^{p-1} \times 0\right)\right) \times I$ taking $S(f) \cap\left(S^{p-1} \times j\right)$ to $\left(S(f) \cap\left(S^{p-1} \times 0\right)\right) \times j$ for $j=0,1$.

Proof. Take the first barycentric subdivision of the triangulation from step (1). Then each simplex $\sigma \subset S(f)$ such that $\sigma \not \subset A_{+} \cup A_{-}$is the join of two simplices $\sigma_{+} \subset$ $A_{+}$and $\sigma_{-} \subset A_{-}$. By Claim 2.4 the polyhedra $\sigma \cap D_{ \pm}^{p}$ are regular neighborhoods of $\sigma_{ \pm}$in $\sigma$. So there is a natural homeomorphism $\sigma \cap\left(S^{p-1} \times I\right) \cong\left(\sigma \cap\left(S^{p-1} \times 0\right)\right) \times I$. Combining such homeomorphisms for all simplices $\sigma \subset S(f)$ such that $\sigma \not \subset A_{+} \cup A_{-}$, we get the required homeomorphism $S(f) \cap\left(S^{p-1} \times I\right) \cong\left(S(f) \cap\left(S^{p-1} \times 0\right)\right) \times I$.

Claim 2.7. There is a homeomorphism $f\left(S^{p-1} \times I\right) \cong f\left(S^{p-1} \times 0\right) \times I$ taking $f\left(S^{p-1} \times j\right)$ to $f\left(S^{p-1} \times 0\right) \times j$ for $j=0,1$.

Proof. (The Alexander trick) By Claim 2.6 the inclusion $i: S(f) \cap\left(S^{p-1} \times I\right) \hookrightarrow$ $S^{p-1} \times I$ is a concordance. Perform an ambient isotopy of $S^{p-1} \times I$ making $i$ an isotopy. Since any isotopy is ambient, there is a homeomorphism $h: S^{p-1} \times I \rightarrow$ $S^{p-1} \times I$ such that $h\left(S(f) \cap\left(S^{p-1} \times I\right)\right)$ is straight, i.e. is equal to $h\left(S(f) \cap\left(S^{p-1} \times\right.\right.$ $0)) \times I$. The required homeomorphism $f\left(S^{p-1} \times I\right) \cong f\left(S^{p-1} \times 0\right) \times I$ is the quotient of $h$. (Analogously to the proof of Claim 2.6 it can be checked that this quotient is well-defined.)

Claim 2.8. There is a homeomorphism of $S^{m-1} \times I$ making $f\left(S^{p-1} \times I\right)$ straight.

Proof. By Claim 2.7 the inclusion $i: f\left(S^{p-1} \times I\right) \hookrightarrow S^{m-1} \times I$ is a concordance. Arguing as in the proof of Claim 2.7 we get the required homeomorphism.

Perform a homeomorphism $S^{m} \rightarrow S^{m}$ extending the one given by Claim 2.8 , The obtained link map is standardized, so for $p \leq q$, Lemma 2.2 is proved.

Proof of Lemma 2.2 for $p=q+1$. The proof is analogous to the proof in the case $p \leq q$; only the cones $B_{ \pm}$and $C_{ \pm}$should be replaced by collapsible polyhedra given by the following claim (cf. [9]).

Claim 2.9. There are collapsible subpolyhedra $B_{ \pm} \subset S^{p}$ and $C_{ \pm} \subset S^{m}$ satisfying Claims 2.4 and 2.5 .

Proof. (The Irwin trick) Let $\bar{B}_{ \pm}$be the polyhedra given by Claim 2.3 , Define $\bar{C}_{ \pm}$ analogously. These polyhedra satisfy all the required properties except $\bar{C}_{ \pm} \cap f S^{p}=$ $f \bar{B}_{ \pm}$. By the inequality $\operatorname{dim}\left(\bar{C}_{ \pm}-f \bar{B}_{ \pm}\right) \cap f S^{p} \leq\left(p-\left[\frac{m+1}{2}\right]+2\right)+p-m \leq 0$ the set $\left(\bar{C}_{ \pm}-f \bar{B}_{ \pm}\right) \cap f S^{p}$ (if nonempty) consists of finitely many points not belonging to $S(f)$. Join each of these points with $\bar{B}_{ \pm}$by a generic arc in $S^{p}$. Let $B_{ \pm}$be the union of these arcs and the cone $\bar{B}_{ \pm}$. Adding appropriate cones over $f\left(B_{ \pm}-\bar{B}_{ \pm}\right)$ to $\bar{C}_{ \pm}$, we get a collapsible polyhedron $C_{ \pm} \subset S^{m}$ such that $\operatorname{dim}\left(C_{ \pm}-\bar{C}_{ \pm}\right) \leq 2$. The polyhedra $B_{ \pm}$and $C_{ \pm}$are the required ones.

The injectivity in Theorem 1.4 is proved by a relative version of the above argument. A standardized link map $f: D^{p} \sqcup D^{q} \rightarrow D^{m}$ is defined as in Definition 2.1. only we fix the standard decomposition of the disc $D^{k}=D_{+}^{k} \cup\left(D^{k-1} \times I\right) \cup D_{-}^{k}$ 
instead of the sphere. Denote by $D_{ \pm}^{k-1}=D_{ \pm}^{k} \cap \partial D^{k}$. Assume that $\partial D_{+}^{k-1}$ is the equator of $\partial D^{k}$.

Lemma 2.10. Suppose that $p \leq q+1, p \leq m-3$ and $2 p+2 q \leq 3 m-5$. Then any generic proper link map $f: D^{p} \sqcup D^{q} \rightarrow D^{m}$ whose restriction to the boundary is a suspension is link homotopic (relatively the boundary) to a standardized link map.

Proof of the injectivity in Theorem 1.4 for $p \leq q$ modulo Lemma 2.10, It suffices to prove that if the suspension of a link map $f_{0}: S^{p} \sqcup S^{q} \rightarrow S^{m}$ is null link concordant, then the link map $f_{0}$ is null link concordant. Take a null link concordance $f$ : $D^{p+2} \sqcup D^{q+1} \rightarrow D^{m+2}$ of $\Sigma f_{0}$. By Lemma 2.10 we may assume that the link map $f$ is standardized.

Push $f D^{q+1}$ along the fibers of $D^{m+1} \times I$ toward $\partial D_{+}^{m+2}$ until it lies in $\partial D_{+}^{m+2}-$ $\partial D^{m+2}$. The restriction of the obtained link map to $\mathrm{Cl}\left(\partial D_{+}^{p+2}-\partial D^{p+2}\right) \sqcup D^{q+1}$ is the required null link concordance of the link map $f_{0}$.

Proof of Lemma 2.10. The proof is analogous to the proof of Lemma 2.2 with the following modifications. Let $\hat{D}^{k}$ be the ball obtained from $D^{k}$ by attaching two cones $C D_{ \pm}^{k-1}$ along $D_{ \pm}^{k-1}$. Let $\hat{f}: \hat{D}^{p} \sqcup \hat{D}^{q} \rightarrow \hat{D}^{m}$ be the obvious extension of the link map $f: D^{p} \sqcup D^{q} \rightarrow D^{m}$. Clearly, it suffices to make the link map $\hat{f}$ standardized (performing homeomorphisms of $\hat{D}^{p}$ and $\hat{D}^{m}$ fixed on the boundary).

(1) Construction of a homeomorphism $\hat{D}^{p} \rightarrow \hat{D}^{p}$ for $p \leq q$. Let $A_{+}$be the union of $\partial D_{+}^{p-1}$ and all simplices of $S(f)$ having dimension not greater than $\frac{1}{2} \operatorname{dim} S(f)$. Let $A_{-}$be the subcomplex dual to $A_{+}$.

Claim 2.11. There are subpolyhedra $B_{ \pm} \subset D^{p}$ collapsible to $B_{ \pm} \cap D_{ \pm}^{p-1}$ and satisfying Claim 2.4.

Proof. Take a generic homotopy $i_{t}: A_{ \pm} \rightarrow D^{p}$ fixed on $A_{ \pm} \cap D_{ \pm}^{p-1}$ such that $i_{0}: A_{ \pm} \hookrightarrow D^{p}$ is the inclusion and $i_{1} A_{ \pm} \subset D_{ \pm}^{p-1}$. Let $B_{ \pm}$be the trace of $i_{t}$.

Take appropriate regular neighborhoods of $B_{ \pm} \cup C\left(B_{ \pm} \cap D_{ \pm}^{p-1}\right)$ in $D^{p} \cup C D_{ \pm}^{p-1}$. Perform a homeomorphism $\hat{D}^{p} \rightarrow \hat{D}^{p}$ fixed on the boundary, taking them to the balls of the standard decomposition of $\hat{D}^{p}$.

Steps (2) and (3) from the proof of Lemma 2.2 are modified analogously.

Thus we have proved Theorem 1.4 for $p \leq q$.

Proof of Theorem 1.4 for $p>q$. The map $\Sigma$ is surjective as the composition

$$
L M_{p, q}^{m} \stackrel{\Sigma^{p-q}}{\longrightarrow} L M_{p, p}^{m+p-q} \stackrel{\Sigma}{\longrightarrow} L M_{p+1, p}^{m+p-q+1} \stackrel{\left(\Sigma^{p-q}\right)^{-1}}{\longrightarrow} L M_{p+1, q}^{m+1},
$$

in which all the maps are well-defined and surjective by the case $p \leq q$ of Theorem 1.4. The injectivity of $\Sigma$ is proved analogously.

\section{Classification of Links}

We prove Theorem 1.1 as follows. First we prove a suspension theorem for links (Theorem 3.1) reducing the classification of links to the classification of disc link maps. Then we simplify the group of disc link maps and find it using the classification of link maps. Formally, Theorem 1.1 follows from 1.4, 3.2, 3.3, 3.5 and the 5-lemma.

Let us introduce some notation. Throughout $₫ 3$ we work in a smooth category. 


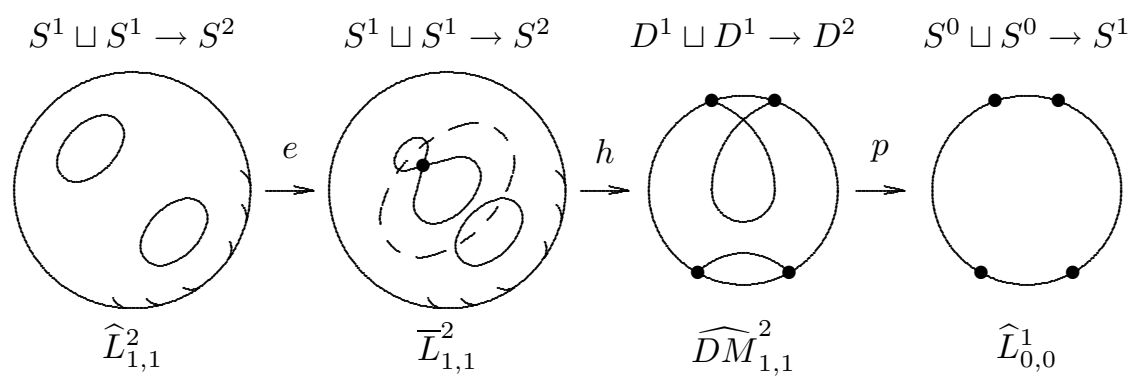

FIgURE 3. Geometric EHP sequence for links

Denote by $\widehat{L}_{p, q}^{m}$ the group of concordance classes of embeddings $S^{p} \sqcup S^{q} \rightarrow S^{m}$, whose restrictions both to $S^{p}$ and to $S^{q}$ are unknotted.

An almost link is a link map $f: S^{p} \sqcup S^{q} \rightarrow S^{m}$ whose restriction to $S^{q}$ is an unknotted embedding. An almost concordance is a link concordance $\left(S^{p} \sqcup S^{q}\right) \times I \rightarrow$ $S^{m} \times I$, whose restriction to $S^{q} \times I$ is a concordance. Let $\bar{L}_{p, q}^{m}$ be the set of almost links up to almost concordance. For $p, q \leq m-3$ this set is a commutative group with respect to the "componentwise connected sum" operation. It is not difficult to see that this group is isomorphic to $\pi_{p}\left(S^{m-q-1}\right)$ (cf. the definition of $\lambda$ below).

A disc link map is a proper link map $f: D^{p} \sqcup D^{q} \rightarrow D^{m}$ whose restriction to $\left(\partial D^{p}\right) \sqcup D^{q}$ is an embedding and $f: \partial D^{p} \rightarrow \partial D^{m}$ is unknotted. A disc link concordance is a proper link concordance $\left(D^{p} \sqcup D^{q}\right) \times I \rightarrow D^{m} \times I$, whose restriction to $\left(\partial D^{p} \sqcup D^{q}\right) \times I$ is a concordance. Let $\widehat{D M}_{p, q}^{m}$ be the set of disc link maps up to disc link homotopy. For $p, q \leq m-3$ it has a natural commutative group structure.

Theorem 3.1 (Geometric EHP sequence for links (A. Skopenkov; cf. [19, 77, Theorem 1.9]; see Figure 31). For $p, q \leq m-3$ there is an exact sequence:

$$
\ldots \longrightarrow \widehat{L}_{p, q}^{m} \stackrel{e}{\longrightarrow} \bar{L}_{p, q}^{m} \stackrel{h}{\longrightarrow} \widehat{D M}_{p, q}^{m} \stackrel{p}{\longrightarrow} \widehat{L}_{p-1, q-1}^{m-1} \longrightarrow \ldots
$$

Proof. Construction of the homomorphisms. Let $e$ be the obvious map. Let $p$ be the "restriction to the boundary" map. The map $h$ is the "cutting" homomorphism defined as follows. Take a generic almost link $f: S^{p} \sqcup S^{q} \rightarrow S^{m}$. Take a generic pair of points $x \in f S^{p}$ and $y \in f S^{q}$ and join them by a path $l$ meeting $f\left(S^{p} \sqcup S^{q}\right)$ only at $\partial l$. Let $\bar{D}^{m}$ be the complement to a small neighborhood of $l$ in $S^{m}$. Denote by $\bar{D}^{p} \sqcup \bar{D}^{q}=f^{-1} \bar{D}^{m}$. Set $h(f)$ to be the restriction of $f$ to a map $\bar{D}^{p} \sqcup \bar{D}^{q} \rightarrow \bar{D}^{m}$.

Proof of the exactness. We have $\operatorname{Im} p=$ Ker $e$ because a link $f: S^{p} \sqcup S^{q} \rightarrow$ $S^{m}$ extends to a disc link map $D^{p+1} \sqcup D^{q+1} \rightarrow D^{m+1}$ if and only if it is almost concordant to a trivial link. We have $\operatorname{Im} h=\operatorname{Ker} p$ because a disc link map $f$ : $D^{p} \sqcup D^{q} \rightarrow D^{m}$ extends without adding new self-intersections to an almost link $S^{p} \sqcup S^{q} \rightarrow S^{m}$ if and only if the restriction of $f$ to the boundary is null-concordant.

To prove Im $e \subset \operatorname{Ker} h$, take a proper embedding $f: D^{p} \sqcup D^{q} \rightarrow D^{m}$. Take a pair of points $x \in D^{p}$ and $y \in D^{q}$. Join $f x$ and $f y$ by an arc $l$ meeting $f\left(D^{p} \sqcup D^{q}\right)$ only at $\partial l$. Let $\bar{D}^{m}$ be a small neighborhood of $l$ in $S^{m}$. Denote by $\bar{D}^{p} \sqcup \bar{D}^{q}=f^{-1} \bar{D}^{m}$. The restriction $f:\left(D^{p}-\bar{D}^{p}\right) \sqcup\left(D^{q}-\bar{D}^{q}\right) \rightarrow\left(D^{m}-\bar{D}^{m}\right)$ is a concordance. By the "concordance implies isotopy" theorem we may assume that this restriction is levelpreserving. Then the Alexander trick shows that the embedding $f: D^{p} \sqcup D^{q} \rightarrow D^{m}$ 
is ambient isotopic to the restriction $f: \bar{D}^{p} \sqcup \bar{D}^{q} \rightarrow \bar{D}^{m}$. The latter embedding is trivial; thus $h \circ e=0$.

To prove Ker $h \subset \operatorname{Im} e$, take $f \in \bar{L}_{p, q}^{m}$ such that $h(f)=0$. By definition, there exists a disc link concordance $c$ between $h(f)$ and an embedding. We may assume that the restriction of $c$ to the boundary is an isotopy. By the isotopy extension theorem [9] it extends to an ambient isotopy of the disc $S^{m}-\bar{D}^{m}$ (from the above definition of the map $h$ ). So $c$ can be extended to an almost concordance between $f$ and a link $f^{\prime} \in \widehat{L}_{p, q}^{m}$. Hence $f=e\left(f^{\prime}\right)$.

Corollary 3.2. $L_{p, q}^{m} \cong C_{p}^{m-p} \oplus C_{q}^{m-q} \oplus \pi_{p}\left(S^{m-q-1}\right) \oplus \widehat{D M}_{p+1, q+1}^{m+1}$ for $p \leq q \leq m-3$.

Proof. By [8, Th. 2.4] we have $L_{p, q}^{m} \cong \widehat{L}_{p, q}^{m} \oplus C_{p}^{m-p} \oplus C_{q}^{m-q}$. So it suffices to show that for $p \leq q$ the homomorphism $e: \widehat{L}_{p, q}^{m} \rightarrow \bar{L}_{p, q}^{m}$ has a right inverse. The required right inverse $\pi_{p}\left(S^{m-q-1}\right) \rightarrow \widehat{L}_{p, q}^{m}$ was constructed in [8, Th. 10.1]: it takes the class of a map $\phi: S^{p} \rightarrow S^{m-q-1}$ to a link $f: S^{p} \sqcup S^{q} \rightarrow D^{q+1} \times S^{m-q-1} \subset S^{m}$ given by the formula $f(x \sqcup y)=\left(\frac{1}{2} x ; \phi x\right) \sqcup(y ; c)$, where $c \in S^{m-q-1}$ is fixed.

Let us simplify the group $\widehat{D M}_{p, q}^{m}$. Define $\overline{D M}_{p, q}^{m}$ to be the group of proper link maps $f: D^{p} \sqcup D^{q} \rightarrow D^{m}$ whose restriction $f: \partial D^{p} \rightarrow \partial D^{m}$ is an unknotted embedding (up to link concordance whose restriction to $\partial D^{p} \times I$ is a concordance).

Lemma 3.3. If $p, q \leq m-3$, then the natural map $\widehat{D M}_{p, q}^{m} \rightarrow \overline{D M}_{p, q}^{m}$ is bijective for $2 p+2 q \leq 3 m-5$ and surjective for $2 p+2 q \leq 3 m-4$.

Proof. The surjectivity. Take a generic link map $f \in \overline{D M}_{p, q}^{m}$. The pair $\left(D^{m}-\right.$ $\left.f D^{p}, \partial D^{m}-f \partial D^{p}\right)$ is $(2 m-2 p-3)$-connected, because $H_{i}\left(D^{m}-f D^{p}, \partial D^{m}-\right.$ $\left.f \partial D^{p}\right) \cong H^{m-i-1}\left(f D^{p}\right)=0$ for $i \leq 2 m-2 p-3$ (because $f D^{p}$ is homotopy equivalent to the mapping cone of the restriction $f: S(f) \rightarrow f S(f)$, having the dimension at most $2 p-m+1$; cf. [6, Lemma 4.2]). Thus by the assumptions $q \leq m-3,2 p+2 q \leq 3 m-4$ and the embedding theorem moving the boundary [9] the restriction $\left.f\right|_{D^{q}}:\left(D^{q}, \partial D^{q}\right) \rightarrow\left(D^{m}-f D^{p}, \partial D^{m}-f \partial D^{p}\right)$ is homotopic to an embedding. So $f$ belongs to the image of the natural map $\widehat{D M}_{p, q}^{m} \rightarrow \overline{D M}_{p, q}^{m}$.

The injectivity. Take a generic link concordance $f:\left(D^{p} \sqcup D^{q}\right) \times I \rightarrow D^{m} \times I$ whose restriction to $D^{q} \times \partial I \cup \partial D^{p} \times I$ is an embedding. It suffices to remove the self-intersection of $D^{q} \times I$ by a link homotopy fixed on $\left(D^{p} \sqcup D^{q}\right) \times \partial I$. It is possible by the following theorem proved similarly to [9], because the pair $\left(D^{m} \times I-f\left(D^{p} \times\right.\right.$ $\left.I), \partial D^{m} \times I-f\left(\partial D^{p} \times I\right)\right)$ is $(2 m-2 p-3)$-connected.

Theorem 3.4 (Embedding theorem moving a part of the boundary). Let $M^{m+1}$, $Y^{m} \subset \partial M$ and $X^{q} \subset \partial D^{q+1}$ be compact manifolds. Let $f:\left(D^{q+1}, X\right) \rightarrow(M, Y)$ be a proper map such that $\left.f\right|_{\partial D^{q+1}-X}$ is an embedding. If $q \leq m-3$ and $(M ; Y)$ is $(2 q-m+2)$-connected, then $f$ is properly homotopic rel $\partial D^{q+1}-X$ to an embedding.

Let us find the group $\overline{D M}_{p, q}^{m}$. Denote by $n=p+q+1-m$. We are going to define a homomorphism $\beta: \overline{D M}_{p, q}^{m} \rightarrow \pi_{n}\left(V_{M+m-p-1, M}\right)$. The following theorem and 5-lemma imply the bijectivity of this homomorphism.

Theorem 3.5 (A. Skopenkov; cf. [13, Th. 3.1], [11, Lemma 5.1], [12, Th. 4.8]). For $p, q \leq m-3$ and $3 p+q \leq 3 m-5$ there is the following diagram with exact 
lines, commutative up to sign:

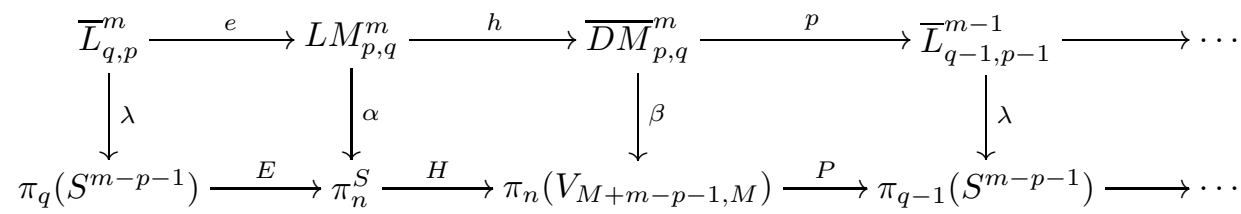

Proof. The top line is defined analogously to Theorem 3.1 (with a similar proof of the exactness). The bottom line is the stable James EHP sequence, for which we use the following geometric construction [15, $\S 1$ and $\S 4$; cf. [10, 25, 44.

Construction of the EHP sequence. Identify the groups $\pi_{q}\left(S^{m-p-1}\right)$ and $\pi_{n}^{S}$ with the groups of framed embeddings and immersions, respectively, of closed $n$ manifolds into $S^{q}$ (up to framed cobordism). A proper immersion is a proper framed immersion of an $n$-manifold into $D^{q}$, whose restriction to the boundary is an embedding. A proper cobordism is a proper framed immersion $c: N^{n+1} \rightarrow$ $D^{q} \times I$, whose restriction to $c^{-1}\left(S^{q-1} \times I\right)$ is an embedding. By [15, Prop. 4.1] we can identify $\pi_{n}\left(V_{M+m-p-1, M}\right)$ with the group of proper immersions up to proper cobordism.

Let $E: \pi_{q}\left(S^{m-p-1}\right) \rightarrow \pi_{n}^{S}$ be the obvious map and let $P: \pi_{n}\left(V_{M+m-p-1, M}\right)$ $\rightarrow \pi_{q}\left(S^{m-p-1}\right)$ be the "restriction to the boundary" map. Let $H: \pi_{n}^{S} \rightarrow$ $\pi_{n}\left(V_{M+m-p-1, M}\right)$ be the cutting homomorphism, defined by removing small discs from an immersed $n$-manifold and the sphere $S^{q}$.

Construction of the vertical homomorphisms. Remove a point from $S^{m}$ and identify the result with $\mathbb{R}^{m}$. For a link map $f: X \sqcup Y \rightarrow \mathbb{R}^{m}$ define the map $\tilde{f}: X \times Y \rightarrow S^{m-1}$ by the formula $\tilde{f}(x, y)=\frac{f x-f y}{|f x-f y|}$. Denote by pr $: X \times Y \rightarrow Y$ the obvious projection.

Definition of $\alpha$ (cf. [12]). Let $f: S^{p} \sqcup S^{q} \rightarrow \mathbb{R}^{m}$ be a generic link map. Take a regular value $v \in S^{m-1}$ of the map $\tilde{f}$. Let $\alpha(f)$ be the cobordism class of the framed immersion pr : $\tilde{f}^{-1} v \rightarrow S^{q}$. (Clearly, $\alpha$ commutes with $\Sigma$; hence by Theorem 1.4 and [12, Th. 2.13] $\alpha$ is an isomorphism for $p, q \leq m-3$ and $2 p+2 q \leq 3 m-5$.)

Definition of $\lambda$ (cf. [8]). Take a generic link map $f \in \bar{L}_{q, p}^{m}$. The complement $S^{m}-f S^{p}$ retracts to a sphere $S^{m-p-1}$ bounding a normal disc to $f S^{p}$. Assume that the sphere $S^{m-p-1}$ is standard. Put the image $f S^{q}$ into the sphere $S^{m-p-1}$ by an appropriate link homotopy rel $S^{p}$. Take a regular value $v \in S^{m-p-1}$ of the map $\tilde{f}$. Let $\lambda(f)$ be the cobordism class of the framed embedding $\operatorname{pr}: \tilde{f}^{-1} v \rightarrow S^{q}$.

Definition of $\beta$ (cf. [13]). Take a generic proper disc link map $f: D^{p} \sqcup D^{q} \rightarrow \mathbb{R}_{+}^{m}$, where $\mathbb{R}_{+}^{m}$ is the upper half-space. By a proper link homotopy, restricting to an isotopy of $\partial D^{p}$, one can put the image $f \partial D^{q}$ into the standard sphere $S^{m-p-1}$. Take a regular value $v \in S^{m-p-1}$ of $\tilde{f}$. Let $\beta(f)$ be the proper cobordism class of the framed proper immersion pr: $\tilde{f}^{-1} v \rightarrow D^{q}$.

Thus the required diagram is constructed. The commutativity up to sign is checked directly.

Remark 3.6. The restriction $2 p+2 q \leq 3 m-6$ in Theorem 1.1 is best possible; the formula fails for $2 p+2 q=3 m-5$. For example, take $m=p+4=4 k-1$, $k \geq 5, q=2 k+1$. Then the group $L M_{p+1, q+1}^{m+1}$ is infinite [13, pp. 755-756]. Thus by Lemma 3.3 and Theorem 3.5

$$
\operatorname{rk} \widehat{D M}_{p+1, q+1}^{m+1} \geq \operatorname{rk} \overline{D M}_{p+1, q+1}^{m+1}>\operatorname{rk} \pi_{p+q+2-m}\left(V_{M+m-p-1, M}\right) .
$$


So by Corollary 3.2 the rank of the left-hand side in the formula of Theorem 1.1 is greater than the rank of the right-hand side.

Remark 3.7. The argument of 8 can be extended to prove Theorem 1.1 at least for $2 p_{1}+2 p_{2} \leq 3 m-7$ (here we use the notation of [8]). Indeed, the only step of that proof in which this restriction is not sufficient is [8, Proposition 10.2]. Since the group $\Lambda_{p_{1}}^{(q)}$ (respectively, $\Pi_{m-2}^{(q)}$ ) is generated by $\theta_{k}\left(i_{1}, i_{2}\right)$ (respectively, $\left[\left[i_{2}, i_{1}\right], i_{2}\right]$ and $\left.\theta_{k+1}\left(i_{1}, i_{2}\right)\right)$ for all $k \geq 0$ such that $k p_{1}+p_{2} \geq(k+1)(m-2)$, the proposition follows. A possible reason why this improvement was not noticed in 8 was that the restriction $2 p_{1}+2 p_{2} \leq 3 m-7$ did not appear there in contrast to $3 p_{1}+p_{2} \leq 3 m-7$.

\section{ACKNOWLEDGEMEnTS}

The author is grateful to A. Skopenkov for constant attention to this work and to the referee for useful suggestions.

\section{REFERENCES}

1. A. Bartels, P. Teichner, All two-dimensional links are null homotopic, Geom. Topol. 3 (1999), pp. 235-252. MR.1714911 (2000i:57043)

2. M. Cencelj, D. Repovs, M. Skopenkov, Homotopy type of the complement to an immersion and classification of embeddings of tori, Russ. Math. Surv. 62:5 (2007), pp. 985-987. MR2373765

3. M. Cencelj, D. Repovs, M. Skopenkov, Knotted tori and the beta-invariant, preprint.

4. P. Eccles, Multiple points of codimension one immersions, Lect. Notes Math. 788, Springer, Berlin (1980), pp. 23-38. MR.585650 (81k:57029)

5. N. Habegger, Knots and links in codimension greater than 2, Topol. 25:3 (1986), pp. 253-260. MR $842424(87 \mathrm{~m}: 57022)$

6. N. Habegger, U. Kaiser, Link homotopy in the 2-metastable range, Topol. 37:1 (1998), pp. 7594. MR 1480878 (98h:57050)

7. A. Haefliger, Differentiable embeddings of $S^{n}$ in $S^{n+q}$ for $q>2$, Ann. Math. (2) 83 (1966), pp. 402-436. MR0202151 (34:2024)

8. — Enlacements de sphères en codimension supérieure à 2, Comm. Math. Helv. 41 (1966-67), pp. 51-72 (in French). MR0212818 (35:3683)

9. J. F. P. Hudson, Piecewise-linear topology, W. A. Benjamin, New York-Amsterdam, 1969. MR0248844 (40:2094)

10. I. M. James, On the iterated suspension, Quart. J. Math., Oxford Ser. (2) 5 (1954), pp. 1-10. MR.0061836 (15:891g)

11. M. Kervaire, An interpretation of $G$. Whitehead's generalization of H. Hopf's invariant, Ann. of Math. (2) 69 (1959), pp. 345-365. MR0102809 (21:1595)

12. U. Koschorke, Link maps and the geometry of their invariants, Manuscripta Math. 61:4 (1988), pp. 383-415. MR952086 (89i:57011)

13. On link maps and their homotopy classification, Math. Ann. 286:4 (1990), pp. 753782. MR1045401 (91b:57031)

14. _ A generalization of Milnor's $\mu$-invariants to higher dimensional link maps, Topology 36:2 (1997), pp. 301-324. MR1415590 (2000a:57063)

15. U. Koschorke, B. Sanderson, Geometric interpretation of the generalized Hopf invariant, Math. Scand. 41 (1977), pp. 199-217. MR0474289 (57:13936)

16. V. Krushkal, P. Teichner, Alexander duality, gropes and link homotopy, Geom. Topol. 1 (1997), pp. 51-69. MR 1475554 (98i:57013)

17. S. Melikhov, Pseudo-homotopy implies homotopy for singular links of codimension $\geq 3$, Uspekhi Mat. Nauk 55:3 (2000), pp. 183-184 (in Russian). MR.1777363 (2001h:57027)

18. L Link concordance implies link homotopy in codimension $\geq 3$, preprint.

19. V. Nezhinsky, A suspension sequence in link theory, Izv. Akad. Nauk 48:1 (1984), pp. 126-143 (in Russian).

20. G.F. Paechter, The groups $\pi_{r}\left(V_{n, m}\right)$. I, Quart. J. Math., Oxford Ser. (2) 7 (1956), pp. 249-268. MR0131878 (24:A1725) 
21. D. Repovs and A. Skopenkov, New results on embeddings of polyhedra and manifolds into Euclidean spaces, Uspekhi Mat. Nauk 54:6 (1999), pp. 61-108 (in Russian). MR.1744658 (2001c:57028)

22. G. P. Scott, Homotopy links, Abh. Math. Sem. Univ. Hamburg 32 (1968), pp. 186-190. MR 0236912 (38:5205)

23. A. Skopenkov, Classification of embeddings below the metastable dimension, submitted, http://arxiv.org/abs/math.GT/0607422

24. _ـ Embedding and knotting of manifolds in Euclidean spaces, in: Surveys in Contemporary Mathematics, N. Young and Y. Choi, eds., London Math. Soc. Lect. Notes 347, Cambridge University Press, Cambridge (2008), pp. 248-342. MR 2388495

25. A. Sjuč, Cobordism groups of l-immersions. II, Acta Math. Acad. Sci. Hungar. 28 (1976), pp. 93-102 (in Russian). MR0440558 (55:13432)

Department of Differential Geometry, Faculty of Mechanics and Mathematics, Moscow State University, 119992, Moscow, Russia

E-mail address: skopenkov@rambler.ru 